Edited by Gerry Edzerdes, Group IS-11

Composition and Layout by Randi Bagley, Croun IS-5

Illustrations by Garry W. Webh, Group N-2

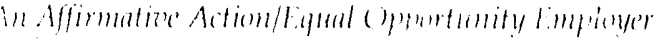

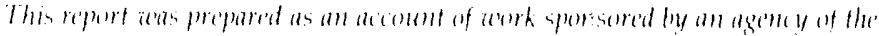

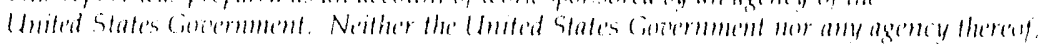

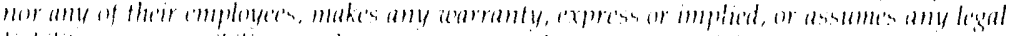

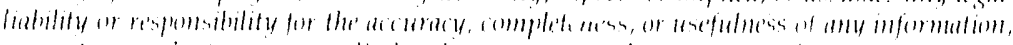

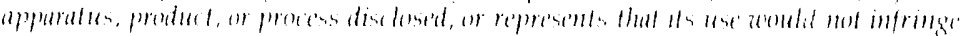

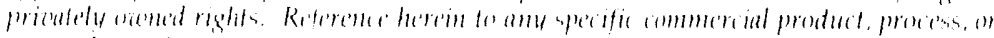

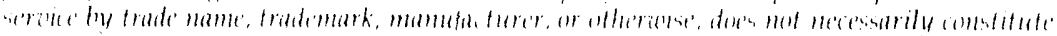

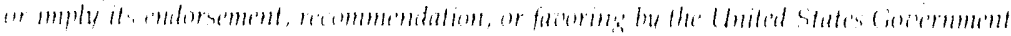

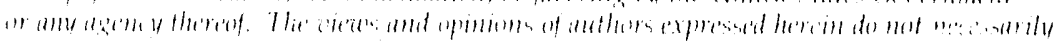

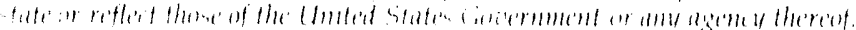


Field-Trial Results for Pre-Flight

Non-Nuclear Verification in

Air Force NELA Flight Tests

Paul E. Fehlau

Hentho

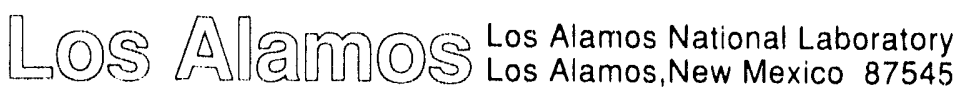

MISTRIBUTION aT THIS DOCUMERT IS UNLIMITED

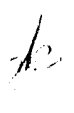




\title{
FIELD-TRIAL RESULTS FOR PRE-FLIGHT NON-NUCLEAR VERIFICATION IN AIR FORCE NELA FLIGHT TESTS
}

by

Paul E. Fehlau

\begin{abstract}
The ruclear-explosive-like assembly (NELA) verification program provides last minute confirmation that NELA test units do not contain nuclear materials when they are launched for flight testing. The program, which is funded through the Weapons Quality Division of DOE's Albuquerque Operations Office, uses instruments developed and maintained by Los Alamos in field operations conducted by Sandia and Air Force personnel. During the period covered here, the goal was to evaluate the instruments in the field and to further develop them for possible routine use by Air Force personnel. A review of more than a year's data from field measurements using two generations of neutron verification instrument shows that the rneasurements agree well with expected results. Reference measurements of real weapons are proportional to similar in-plant confirmation measurements by Pantex Plant personnel using different, less portable instruments. Results for NELA Joint Test Assembly verification have all been close to background results and well below the corresponding result. 3 for real weapons. The two instrument failures that occurred were recognized at the time of failure. The few personnel failures that occurred were a result of insufficient time for training and a lack of comprehensive written procedures.
\end{abstract}

\section{INTRODUCTION}

Special nuclear material (SNM) confirmation techniques examine some attribute of a material or item to assure that the material or item is the expected one. When the attribute can be quantified, the confirmation procedure is called verification and involves measuring the attribute for comparison to a recorded value for the same or similar item. Many confirmation procedures are used during the manufacture, storage, transportation, and deployment of a nuclear weapon. Similar nuclear-explosive-like assemblies (NELAs) undergo the same and possibly more confirmation steps to ensure that they are indeed 
non-nuclear and do not contain SNM. The non-nuclear verification instrument discussed here is a neutron detection instrument that is sensitive, readily portable, and easily used to make a final, non-nuclear verification of a NELA Joint Test Assembly (JTA) just before launch.

For many years, pre-flight verification at a launch site was possible only if someone from the DOE Pantex Plant was present with a set of the Plant confirmation equipment, which measures characteristic neutron and gamma-ray emissions of SNM. More recently, some segments of the military adopted pre-flight verification with portable radiation measurement equipment as part of launch safety procedures. A few years ago, the Weapons Quality Division of DOE's Albuquerque Operations Office began to encourage universal pre-flight verification of JTAs at the launch site using radiation measurements. At the time, Los Alamos had already developed a hand-held instrument for radiation measurement that could be modified for making neutron or gamma-ray confirmation measurements. Los Alamos commercialized the concept in two types of instrument ${ }^{1,2}$ : (1) the Jomar Systems* JHH-01 gamma-ray verification instrument; and (2) a neutron verification instrument now available in three commercial versions, the TSA Systems ${ }^{* * *}$ PR,M-470N and NNV-470, and the Jomar JHH-22 neutron verification instruments.

The Jomar JHH-01 gamma instrument and a modified, neutron health-physics instrument were first used to evaluate verification with hand-held instruments at Pantex; the instruments produced results that were proportional to the results obtained with the routinely-used Pantex confirmation instruments. When the Jomar JHH-22 became available, the neutron and gammu-ray instruments were used to begin an Air Force pre-flight verification program, using Sandia and Air Force personnel to make verification measurements. Early experience with the two types of instrument led to selecting the neutron instrument for routine use. The basis for the choice of the neutron instrument was that it is less complex, easier to maintain, and simpler to operate, making it more suitable for routine field use. A modified version of the reutron irstrument was designed and renamed the TSA NNV-470 to reflect its use for non-nuclear verification. The modified design incorporates changes to meet the perceived needs of the Air Force application, such as being operable in the dark while wearing heavy gloves. The program continued with the JHH-22 and PRM-470N instruments, and the new NNV-470 was included when it became available. 'This report examines the results of the first extensive period of neution verification of JTAs by Sandia and Air Force personnel.

\section{THE NEUTRON VERIFICATION INSTRUMENT}

Neutron emission is the radioactive attribute of plutonium that offers the most convenient means to confirm its presence. Neutrons can easily penetrate most materials to give a signature of plutonium presence at the surface of an assembly or othor packagre.

:Jomar Systems, Inc, 110 Eastgate Drive, Los Alamos, NM 87544 (505) 662-9811.

TSA Systems, Ltd., 1820 Delaware Place, Longmont, CO 80501 (303) 651-61.17. 
Neutron backgrounds are naturally low, and sources of neutrons other than plutonium are unlikely to be present at Air Force launch sites. Hence, a significant number of neutrons detected during verification would most likely indicate the presence of plutonium and, therefore, a real weapon instead of a JTA.

Figure 1 shows the Los Alamos neutron verification instrument in its latest commercial version, the NNV-470. It uses a moderated, $2.5-\mathrm{cm}$-diam, enriched lithium iodicle $\left[{ }^{6} \mathrm{LiI}(\mathrm{Eu})\right]$ scintillator to convert gamma rays and neutrons to light, which is then converted to current and voltage pulses. The instrument has a voltage-level discriminator to select the large pulses produced by neutrons for measurement and an LCD to display the measurement results. The measurement result (the number of neutrons counted by the instrument) determines whether or not plutonium is present.

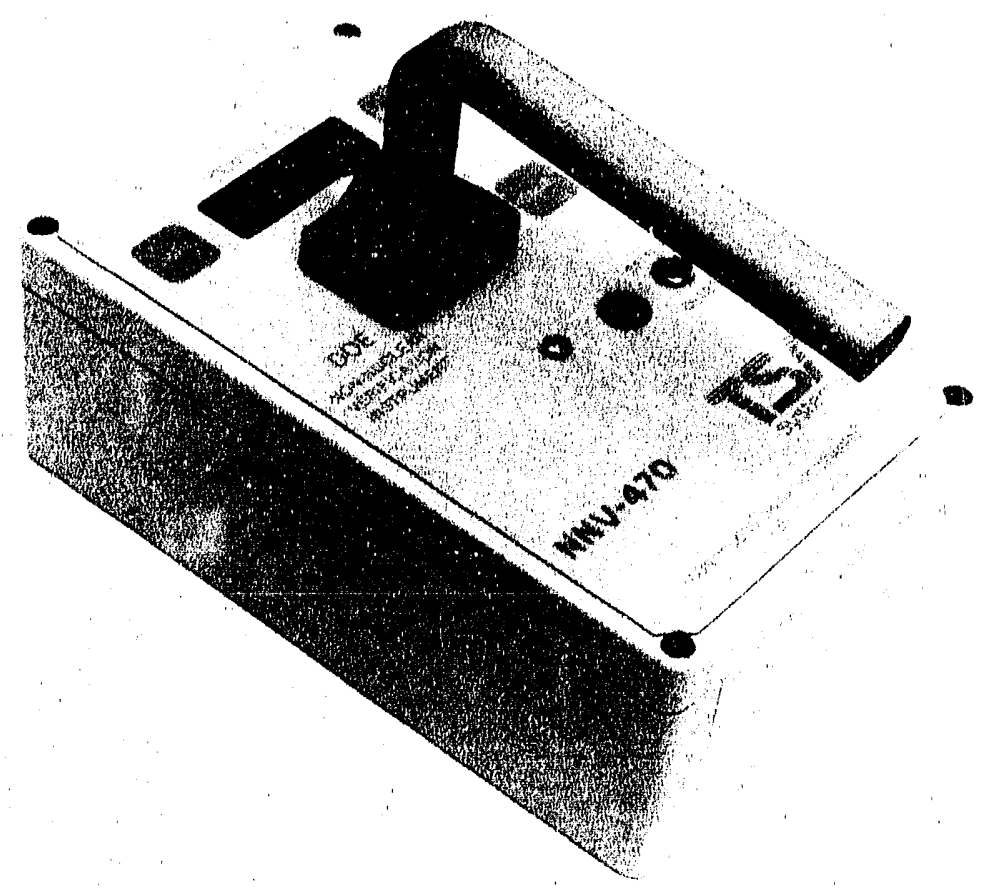

Fig. 1. The TSA Systems NNV-470 is a hand-held instrument designed to detect and measure neutrons in the mixed radiation fields that may be present from real weapons or NELAAs.

For JTA verification, the instrument measures neutrons for a preselected time period of $20 \mathrm{~s}$. Non-nuclear verification measurement results are usually well below 20 counts in a 20-s gross measurement. As each result is displayed, the instrument beeps and starts a new measurement while holding the latest result in its display. This permits making 
several measurements and recording the results during confirmation. Verifying normal operation of the instrument and measuring background intensity are also part of confirmation. If a neutron source for verifying operation is not available, the instrument has a second check mode that can be used. The check uses a second voltage-level discriminator set very low so that gamma rays can also be detected.

\section{MAKING VERIFICATION MEASUREMENTS}

Verification measurements are usually made at the position of maximum respon ie on each item. The instrument is placed at that position with its bottom in contact with or as close as rules allow to the surface of the JTA. In many cases, a felt spacer is used to avoid contact. The following is a sequence of steps for making verification measurements.

1. Before starting measurements, switch the instrument on long enough to begin displaying numerical results, then check it for normal operation with a radiation source.

2. Once normal operation is verified, measure and record background at a short distance ( 3 to $6 \mathrm{ft}$ ) from the item to be verified and with the operator's body between the item and the instrument. Other shielding by objects or people around the instrument is not permitted, nor are neutron sources permitted to be nearby.

3. Place the instrument and properly orient it at the measurement position while observing and recording three count values.

4. Repeat the background and source measurements to be sure nothing about the instrument or environment has changed.

5. Calculate the net verification result by subtracting the average background from before and after the verification measurement from the average of the verification measurements. Net results of 20 or less indicate a non-nuclear item.

6. If the results appear normal, this completes verifiation. If there is an unexpected result, all the measurements in the sequence can be repeated, and a second instrument is normally available to provide an independent verification.

\section{THE FIELD REFERENCE MEASUREMEN'TS}

Verification compares a measurement result with a predetermined reference measurement result. Most reference measurements for the Air Force program were obtained at the weapon stations of the Air Force bases visited for pre-flight JTA verification. Other reference measurements were made whenever an opportunity arose, which sometimes led to measuring real weapons used in the other armed services. The reforence measurements included bare warhead measurements, measurements of warheads in shipping containers, and measurements of warhoads loaded in missiles and launchers. 
We plotted the reference measurement results against values for the same weapons from the data base of Pantex confirmation measurements. The plot in Fig. 2 gives the average measurement value for each case; error bars represent the experimental standard deviation (when three or more measurements are available).

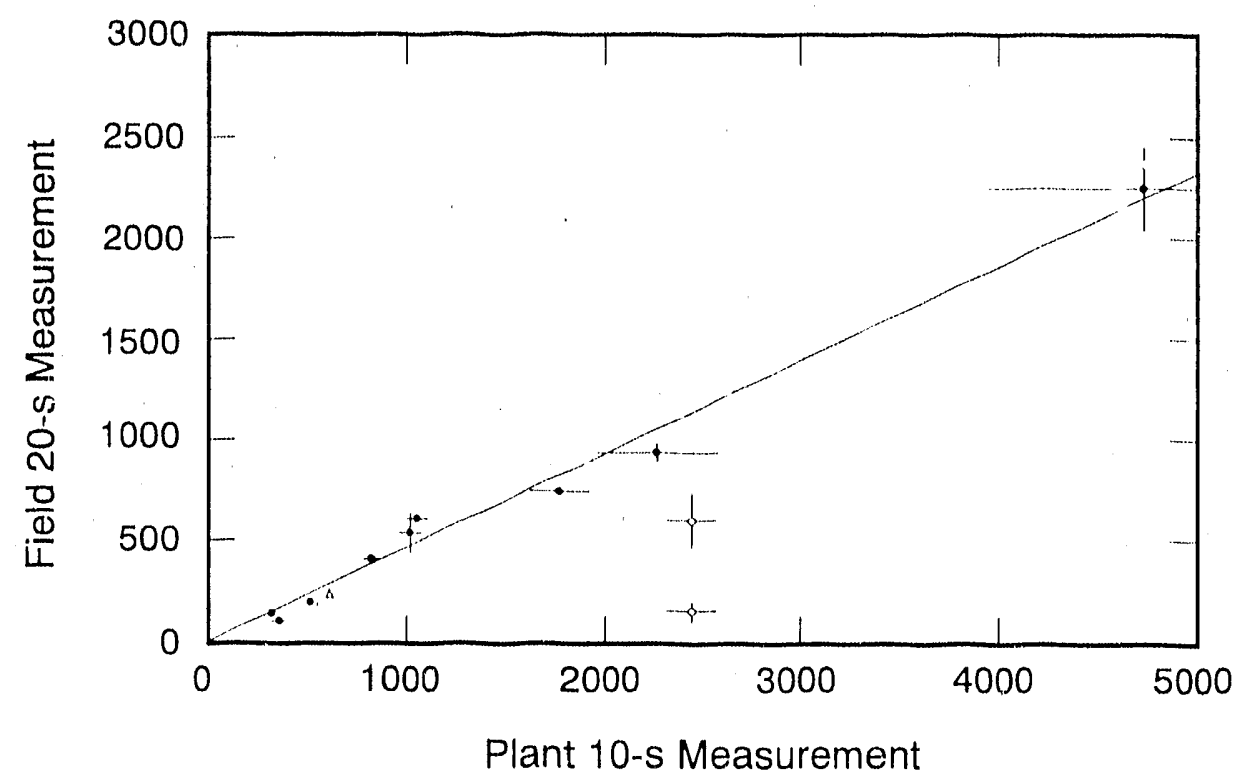

Fig. 2. The field results obtained with the hand-held neutron verification instrument (y-axis) measuring real weapons show good proportionality with the corresponding Pantex confirmation results ( $x$-axis). The outlying open-symbol points are for warheads in shipping containers or launch vehicles.

In Fig: 2, the crror bars for the Pantex plant measurements are often much larger than the error bars for the field measurements. The reason for this is that the plant background can be highly variable because of the presence of other nearby weapons; background variation is usually less during field measurements.

The dark circles plotted in Fig. 2 represent results for bare warheads. The lowest point plotted on the line has its lower error bar at about 90 counts (per $20 \mathrm{~s}$ ). Hence, the decision point of 20 mentioned earlier reasonably separates real weapons from J'T'As. The open triangle represents a result for a warhead in a shipping container plotted against the corresponding Pantex result. The open diamonds are for a warhead in a missile and launcher plotted against the Pantex result for the bare warhead. These are all above the lowest point plotted on the line.

\section{THE FIELD BACKGROUND AND J'TA MEASUREMEN'TS}

In the preceding section, the reference measurements turned out to be well above the number 20 used as the decision threshold for verifying a JTA. Hence, the miss probability for detecting a real weapon should be small. The field background and JTA measurements 
give evidence that the nuisance-alarm probability of misidentifying a true J'TA as a real weapon is also small. Table I summarizes background measurements obtained while verifying different types of JTA. The largest background measurement result was 5 , and it occurred only once in 454 background measurements (usually six but sometimes only three background measurements were made during each verification). Table II summarizes the corresponding JTA verification measurements. The largest gross JTA result was 7, and it occurred only twice in 257 verification measurements (usually 3 JTA measurements were made during each verification). It seems unlikely that any JTA verification result would exceed 20 , even if the background were not subtracted for some reason.

Table I. Summary of Background Results

\begin{tabular}{|c|c|c|c|}
\hline $\begin{array}{l}\text { Number of } \\
\text { Measurements }\end{array}$ & Average (counts) & Std Dev (counts) & Results Equal to 5 \\
\hline 78 & 0.29 & 0.68 & 0 \\
\hline 57 & 0.78 & 0.93 & 0 \\
\hline 24 & 0.96 & 1.4 & 0 \\
\hline 76 & 0.65 & 0.84 & 0 \\
\hline 24 & 0.38 & 0.65 & 0 \\
\hline 81 & 0.77 & 0.84 & 1 \\
\hline
\end{tabular}

Table II. Summary of JTA Gross Results

\begin{tabular}{|c|c|c|c|}
\hline $\begin{array}{l}\text { Number of } \\
\text { Measurements }\end{array}$ & Average (counts) & Std Dev (counts) & Results Equal to 7 \\
\hline 58 & 0.82 & 1.45 & 0 \\
\hline 43 & 0.86 & 1.13 & 0 \\
\hline 9 & 0.78 & 0.44 & 0 \\
\hline 39 & 1.80 & 1.53 & 1 \\
\hline 12 & 1.1 & 1.0 & 0 \\
\hline 42 & 0.78 & 0.93 & 0 \\
\hline 54 & 1.74 & 1.56 & 1 \\
\hline
\end{tabular}


In the tables, the averages of the JTA measurement results are often larger than the background result averages. However, the standard deviations are large enough to give little confidence in the difference. But if there were a true difference in the averages, it could result from measuring; cosmic-ray-produced neutrons in the JTA or neutrons emitted by any depleted uranium in the J'TA, or it could be an artifact, possibly one caused by preferentially recording larger results when measuring a J'TA.

\section{SUMMARY OF THE MEASUREMENT RESULTS}

From the data available, it appears that the threshold of 20 can be used to separate JTAs from real warheads without a serious chance of missing a real warhead or miside tifying a JTA. Using the single value of 20 eliminates the need for transferring a catalog of specific results for each warhead to the launch site. The single value also decreases the chance of making one type of error: using the wrong reference value for the particular JTA being verified.

A word of caution about the range of reference values: there is no way of knowing whether the reference measurement that would give the lowest possible result for a real. weapon has been made. There may be other circumstances in the future for which the distance between the verification instrument and a real weapon or JTA could be greater than we have experienced so far. In that case, the corresponding reference measurement value could be significantly lower than 90. Other containers or missiles could also significantly reduce reference measuremeni values. We must continue to accumulate these results in the field to cover new situations.

We must also continue making and recording source, barkground, and JTA measurements to serve as a form of measurement control that will allow us to be certain that instruments or measurement techniques do not change and begin to give numorical results that are different than we have thus far experienced.

\section{SUMMARY OF O'THER RESULTS}

Two instruments failed during the time period examined here (the failures were recognized at the time that they occurred). One failure was in the first JHH-22 prototype unit, in which the mechanical attachment of the scintillator to the photomultiplier failed. The second failure was in a PRM-470N battery pack, which might normally be replaced periodically as part of a maintenance program.

Shortcomings in the performance of the individuals who conducted the verification measurements were also infrequent 'Those that occurred were caused by a lack of sufficient training time. In some cases, a lack of familiarity with the procedures led to not recording enough information to avoid later confusion. Typical omissions were not recording the instrument serial number, the J'I'A or weapon identification number, or the information on the exact position at which the verification measurement was made. 
The instruments were occasionally returned to Los Alamos for recalibration during the period reviewed here. During recalibration, the instruments measured a test source assembly, designed to give a net verification result of about 100 . The net test results for different instruments ranged from 83 to 120. The range for net test source results by particular instruments at different times was much smaller. Hence, we can expect the variation in verification results for different instruments (or the same instrument at different times) to be relatively small compared to the gaps between the decision threshold of 20 and the lowest reference result for a real weapon or an average net JTA measurement result.

\section{ACKNOWLEDGMENTS}

David Millegan, LANL, was responsible for maintaining and calibrating the verification instruments. Karen Melgaard and Roger Case, SNL, assisted with recalibration on occasions when it was necessary to process a large batch of instruments in one day, Roger Case also collated the field data, making it much easier to summarize and evaluate.

\section{REFERENCES}

1. P. E. Fehlau, "Rugged, Lightweight, and Long-Operating Hand-Held Instruments for Neutron and Gamma-Ray Verification Measurements," Proc. 22nd Midyear Topical Meeting of the Health Physics Society on Instrumentation, San Antonio, Texas, December 4-8, 1988. Los Alamos National Laboratory document LA-UR-88-2780.

2. P. E. Fehlau and G. Wiig, "Stabilized, Hand-Held, Gamma-Ray Verification Instrument for Special Nuclear Materials," IEEE Trans. Nucl. Sci. NS-36, 1160-1165 (February 1989). Los Alamos National Laboratory document LA-UR-88-3673. 

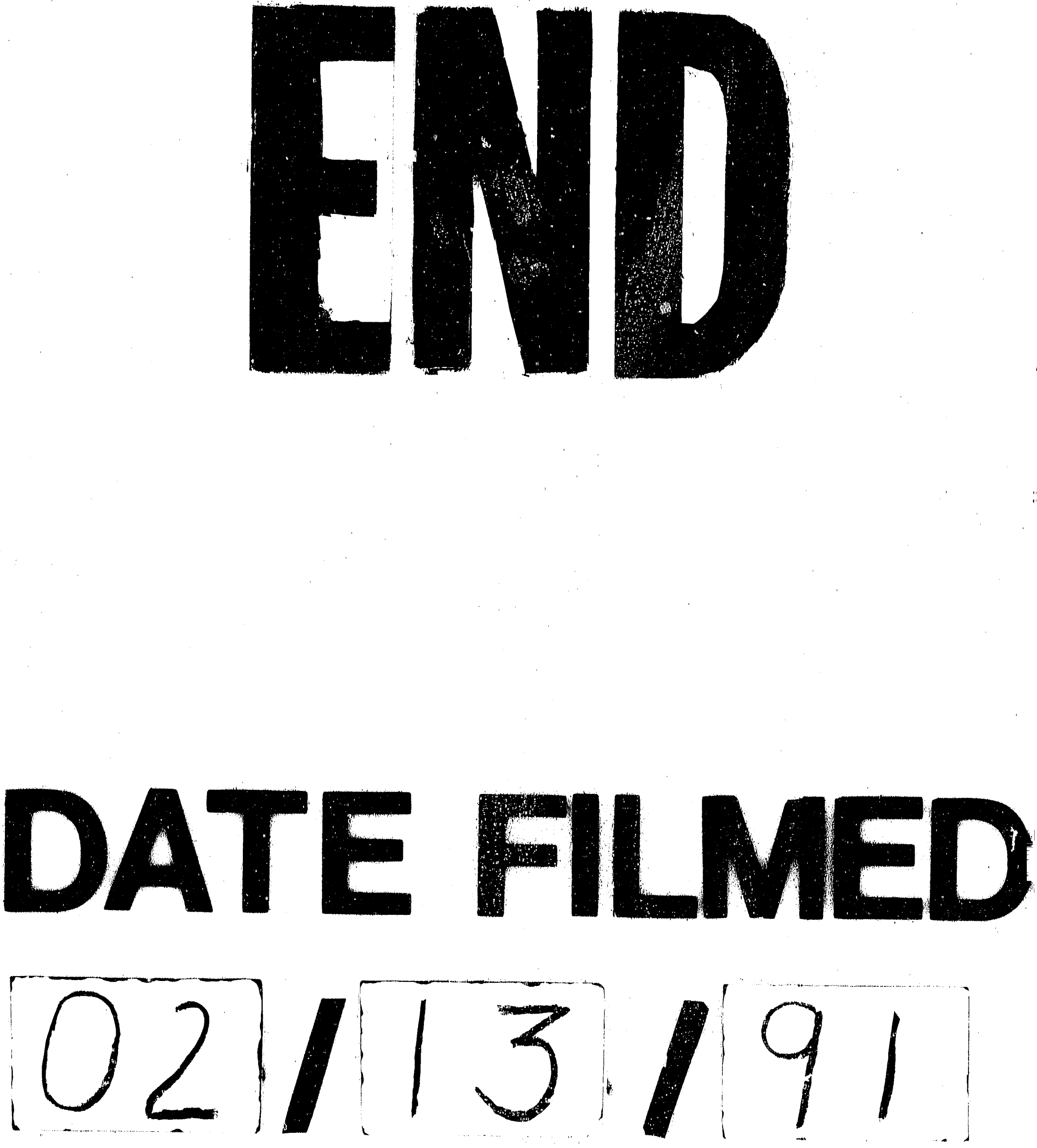
